5 Pinming LIU, Yan GAO, Xiangyang FU, Junhao LU, Ying ZHOU, Xianglong WEI et al. Pump models assessed by transoesophageal echocardiography during cardiopulmonary resuscitation. Chin Med J 2002; 115(3): 359-363.

6 Kadrmas EF, Pach JM. Vitreous haemorrhage and retinal vein rupture. Am J Ophthalmol 1995; 120(1): 114-115.

7 Jones WL. Valsalva maneuver induced vitreous haemorrhage. J Am Optom Assoc 1995; 66(5): 301-304.

8 Konotey-Ahula F. Valsalva vitreous haemorrhage and retinopathy in sickle cell haemoglobin C disease. Lancet 1997; 349(9067): 1774

JR Cameron, P Cackett, A Tey and H Bennett

Princess Alexandra Eye Pavilion, Chalmers Street, Edinburgh EH3 9HA, Scotland, UK

Correspondence: JR Cameron,

Tel: + 44131536 3778;

Fax: + 441315363897

E-mail: j.cameron@doctors.org.uk

Declaration: No funding was received in the preparation of this manuscript. The work has not been presented at any meeting.

Eye (2006) 20, 1317-1319. doi:10.1038/sj.eye.6702184; published online 18 November 2005

Sir,

\section{An unusual case of proptosis}

We report a rare case of orbital xanthoma in a 38-year-old man presenting as droopy eyelid and proptosis. The only clue to the diagnosis was the presence of eyelid xanthelasma.

\section{Case report}

A previously well 38-year-old Bangladeshi labourer presented with a 2-month history of worsening right ptosis, proptosis, and dull ache over the forehead (Figure 1). Examination revealed a right non-axial proptosis which measured $23 \mathrm{~mm}$ on Hertel's exophthalmometer (compared with $21 \mathrm{~mm}$ on the left). The right palpebral fissure measured $5 \mathrm{~mm}$ (compared with $7 \mathrm{~mm}$ on the left). The right upper eyelid had an area of xanthelasma in the nasal aspect; otherwise, it is not erythematous or tender to palpation. The ocular movement was normal except for dull ache and slight restriction on upgaze. The vision was normal in both eyes. An urgent CT scan revealed a mass which appeared to involve the superior rectus and levator superioris complex (Figure 2). A possible diagnosis of myositis was made and he was started on oral $100 \mathrm{mg}$ diclofenac b.d. The blood tests for autoimmune antibodies, ESR, and thyroid function tests were normal. The only abnormal result was raised cholesterol level of $6.2 \mathrm{mmol} / 1$. There was no clinical improvement after 2 weeks. A MRI scan was performed to elucidate the lesion. This showed the mass to be distinct from the superior rectus and appeared to involve only the levator superioris (Figure 3). An open biopsy through the skin crease revealed an uncapsulated orange coloured lesion between the orbicularis oculi and the orbital septum with infiltration of the levator aponeurosis (Figure 4). The lesion had a rubbery consistency and extended posteriorly beneath the orbital roof. The mass was removed en bloc. It had a uniform yellow surface

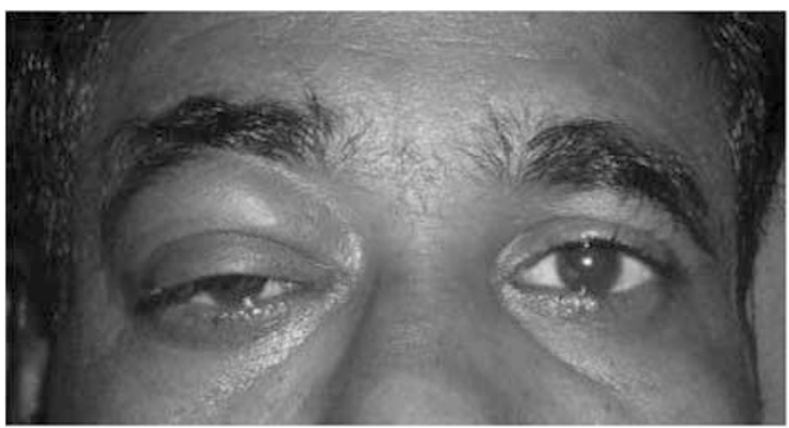

Figure 1 A 38-year-old Bangladeshi labourer with right eye ptosis and proptosis.

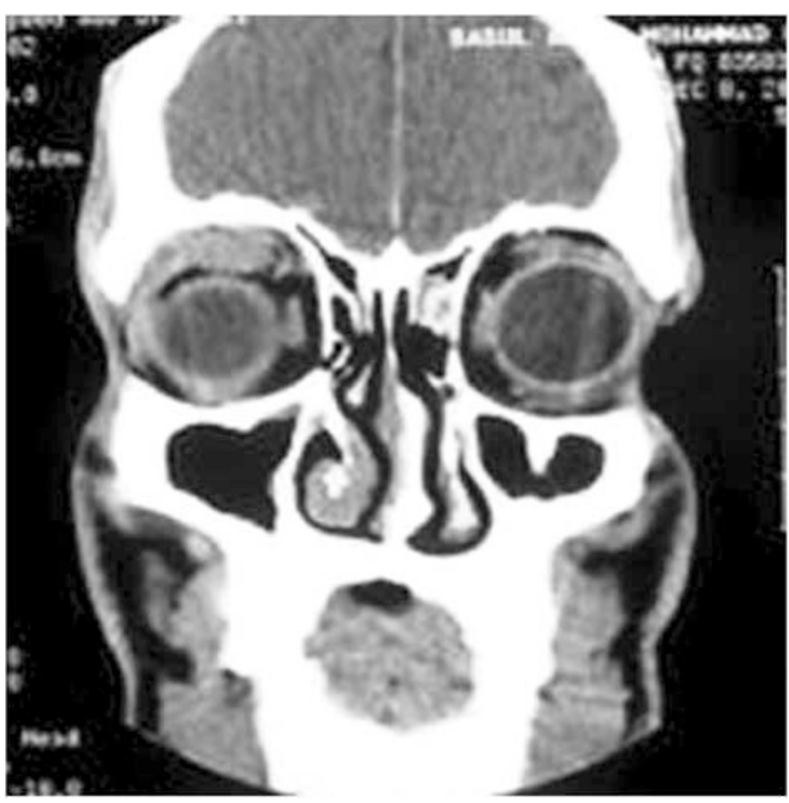

Figure 2 A CT coronal scan of the orbit revealed a mass at the right eye superior rectus and levator superioris complex. 


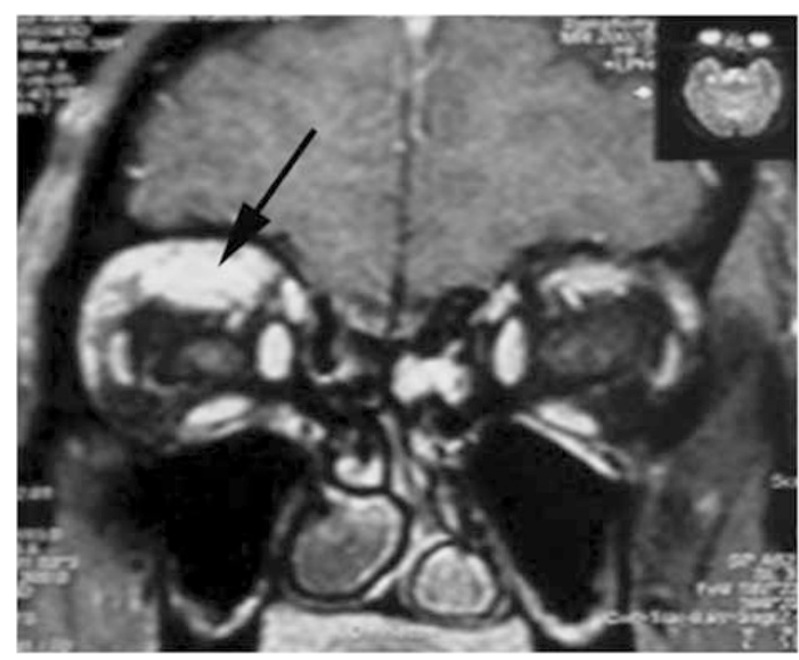

Figure 3 A MRI coronal scan of the orbit showed the mass to be distinct from the right superior rectus and appear to involve only the right levator superioris.

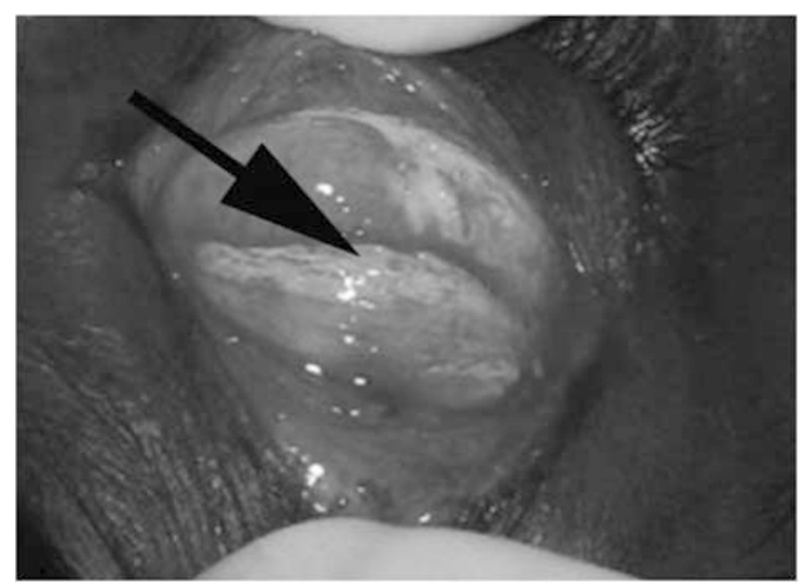

Figure 4 An open biopsy through the skin crease of the right eye revealed an uncapsulated orange coloured lesion between the orbicularis oculi and the orbital septum with infiltration of the levator aponeurosis.

measuring $2.1 \times 1.5 \times 1.1 \mathrm{~cm}$. Postoperatively, there was a marked improvement of the proptosis. Histological report revealed a fibroadipose lesion made up of large number of macrophages with foamy cytoplasm and eccentric nuclei. There were no Touton's giant cells seen to suggest the diagnosis of xanthogranuloma. The final pathology diagnosis was orbital xanthoma (Figure 5).

\section{Comment}

Xanthoma occurring within the orbit is extremely rare. There have been only a few reports in the literature. ${ }^{1-3}$ They are typically found in the subcutaneous tissues

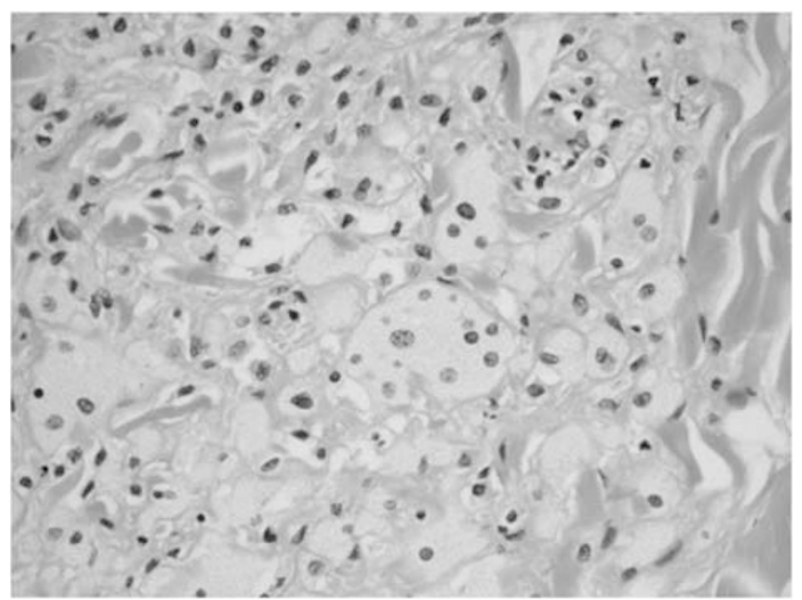

Figure 5 Histology slide showing orbital xanthoma.

usually along the Achilles tendon and extensor tendons of the hand of patients with hyperlipidaemia. They are believed to develop as a result of leakage of lipids from the blood vessels into the surrounding tissue. The lipids were subsequently phagocytosed by macrophages. As the cholesterol is not degraded, the macrophages have foamy cytoplasm. Extracellular cholesterol can induce chronic inflammatory reaction which may account for the dull ache experienced by our patient. Giant cells similar to that seen in chalazion may occur in response to the inflammation and fibrosis may develop. The condition needs to be differentiated from two other conditions: xanthogranuloma and necrobiosis xanthoma. In the former, Touton's cells are found histologically and in the later the blood tests show dysproteinaemia.

On the CT and MRI scans, xanthoma could not be differentiated from other orbital benign tumours such as frontal mucocele, epidermoid cyst, dermoid cyst,

haemangioma, meningioma, Langerhans cell histiocytoma, and fibrous dysplasia. ${ }^{4}$ In cases of chronic inflammation, the clinical features may be confused with pseudotumour leading to unnecessary investigations for autoimmune diseases. Biopsy is the only certain mean of diagnosing orbital xanthoma.

In summary, as orbital xanthoma cannot be diagnosed with certainty either clinically or by radiological imaginations, this unusual condition should be included in the differential diagnosis of proptosis in patients with eyelid xanthelasma or hyperlipidaemia.

\section{References}

1 Kodsi S, Valderrama E. Orbital xanthoma in a 9-month-old infant. Am J Ophthalmol 2001; 131(1): 150-151.

2 Ohta M, Suzuki Y, Sawada M. Bilateral tumor-like invasive xanthelasma palapebrarum in the superior palpebra. Ophthal Plast Reconstr Surg 1996; 12(3): 196-198. 
3 Mohan H, Sen DK, Chatterjee PK. Localized xanthomatosis of orbit. Am J Ophthalmol 1970; 69(6): 1080-1082.

4 Matoba M, Tonami H, Kuginuki M, Yamamoto I, Akai T, Iizuka H. CT and MRI findings of xanthoma in the orbitofrontal region. Radiat Med 2004; 22(2): 116-119.

\section{RSH Chung, A Wagle and CN Chua}

The Eye Institute at Alexandra Hospital, 378

Alexandra Road, National Healthcare Group, Singapore 159964

\author{
Correspondence: RSH Chung \\ Tel: + 6563793510 ; \\ Fax: + 6563793618 \\ E-mail: Ronald_Chung2001@Yahoo.com
}

Proprietary/Financial interest: None

Eye (2006) 20, 1319-1321. doi:10.1038/sj.eye.6702185; published online 9 December 2005

Sir,

Resolution of cystoid macular oedema after retinal detachment repair: is intravitreal triamcinolone useful?

Poor functional visual outcome after successful retinal detachment (RD) repair is mainly owing to photoreceptor cell apoptosis. ${ }^{1}$ Clinical appearance of the macula in the majority of these patients is normal, but a proportion have anatomical changes observable clinically. ${ }^{2}$ More recently, ocular coherence tomography (OCT) has helped in revealing residual subfoveal fluid accumulation not visible clinically or on fluorescein angiography, ${ }^{3}$ more frequent after scleral surgery. ${ }^{4}$ OCT has also been helpful in showing postoperative cystoid macular oedema (CMO), responsive for the limited visual improvement in a small number of patients. The exact etiopathogenic mechanism of CMO after RD is unclear, but ocular inflammation may play a role, especially after the trauma of cryotherapy, scleral buckle, and subretinal fluid (SRF) drainage. CMO incidence, in phakic eyes, has been reported around 25-30\% after cryotherapy and scleral explant, ${ }^{5}$ and $8 \%$ after pneumatic retinopexy. ${ }^{6}$ It has not been related to preoperative macular status or duration of the RD. ${ }^{7} \mathrm{CMO}$ spontaneous resolution has been described in up to $76 \%$ of cases, ${ }^{5}$ within 2 years postoperatively. Other series, though, report a more bleak evolution of the condition. ${ }^{8}$ Different treatments have been described, but none with great success. Topical and systemic non-steroidal antiinflammatory drugs and steroids have been used. There are anecdotic reports of response to acetazolamide. ${ }^{9}$
A 78-year-old male presented to our Primary Care Department with complaints of floaters for the past week, and decreased central vision for the last $48 \mathrm{~h}$. He underwent uneventful phacoemulsification and posterior chamber intraocular lens implant 3 years ago, but otherwise he had no other past ocular history. On examination, visual acuity was 6/36 and fundoscopy showed superotemporal bullous RD involving his macula, for which he underwent pars plana vitrectomy, cryoretinopexy, and $12 \% \mathrm{C}_{3} \mathrm{~F}_{8}$ gas endotamponade.

Immediate postoperative period was anodyne, with complete flattening of the retina. At 4 months after successful repair, though, best-corrected visual acuity (BCVA) was 6/18. Anterior segment was quiet with pseudophakia and fundoscopy revealed cystoid macular changes. OCT confirmed this clinical finding and also showed no presence of residual SRF (Figure 1). The patient was started on topical ketorolac and prednisolone, and 1 month later received $4 \mathrm{mg}$ intravitreal triamcinolone acetonide (IVTA). OCT performed 2, 6, and 12 months later, still showed cystoid spaces in the macula and BCVA remained unchanged (Figure 2). At 15 months after IVTA, OCT showed total resolution of the CMO (Figure 3). Visual acuity, at this stage, had returned to pre-RD levels, with BCVA of 6/6.

There are several reports of successful treatment of uveitic, pseudophakic, and diabetic CMO with IVTA. However, no reference to $\mathrm{CMO}$ after RD treated with IVTA could be found in a PubMed literature search. There is a rationale for using steroids in its management, as increased prostaglandin levels in the retina and uvea and increased prostacyclin and thromboxane $\mathrm{A}_{2}$ derivatives in the SRF have been reported. ${ }^{10}$ All these mediators increase perifoveal microvascular permeability by disrupting capillary tight junctions,

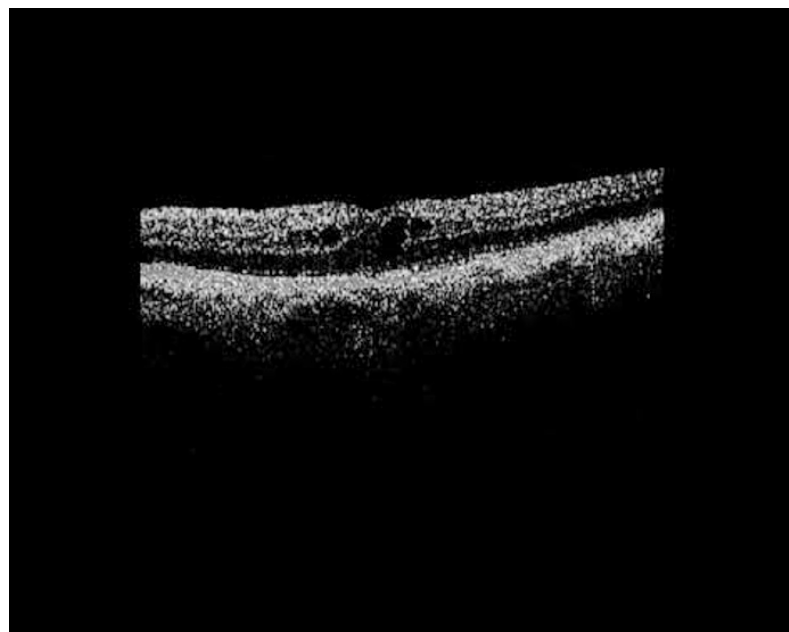

Figure 1 OCT scan 4 months after successful retinal detachment repair, showing cystoid macular oedema. 